\title{
Limits on Spin-Independent Interactions of Weakly Interacting Massive Particles with Nucleons from the Two-Tower Run of the Cryogenic Dark Matter Search
}

\author{
D. S. Akerib, ${ }^{2}$ M. J. Attisha, ${ }^{1}$ C. N. Bailey, ${ }^{2}$ L. Baudis, ${ }^{11}$ D. A. Bauer, ${ }^{3}$ P. L. Brink, ${ }^{7}$ P. P. Brusov, ${ }^{2}$ R. Bunker, ${ }^{9}$ B. Cabrera, ${ }^{7}$ \\ D. O. Caldwell, ${ }^{9}$ C. L. Chang, ${ }^{7}$ J. Cooley, ${ }^{7}$ M. B. Crisler, ${ }^{3}$ P. Cushman, ${ }^{6}$ M. Daal, ${ }^{8}$ R. Dixon, ${ }^{3}$ M. R. Dragowsky, ${ }^{2}$ \\ D. D. Driscoll, ${ }^{2}$ L. Duong, ${ }^{6}$ R. Ferril, ${ }^{9}$ J. Filippini, ${ }^{8}$ R. J. Gaitskell, ${ }^{1}$ S. R. Golwala, ${ }^{12}$ D. R. Grant, ${ }^{2}$ R. Hennings-Yeomans, ${ }^{2}$ \\ D. Holmgren, ${ }^{3}$ M. E. Huber ${ }^{10}$ S. Kamat, ${ }^{2}$ S. Leclercq, ${ }^{11}$ A. Lu, ${ }^{8}$ R. Mahapatra, ${ }^{9}$ V. Mandic, ${ }^{8}$ P. Meunier,${ }^{8}$ N. Mirabolfathi, ${ }^{8}$ \\ H. Nelson, ${ }^{9}$ R. Nelson, ${ }^{9}$ R. W. Ogburn, ${ }^{7}$ T. A. Perera,${ }^{2}$ M. Pyle, ${ }^{7}$ E. Ramberg, ${ }^{3}$ W. Rau, ${ }^{8}$ A. Reisetter, ${ }^{6}$ R. R. Ross, ${ }^{4,8, *}$ \\ B. Sadoulet, ${ }^{4,8}$ J. Sander, ${ }^{9}$ C. Savage, ${ }^{9}$ R. W. Schnee, ${ }^{2}$ D. N. Seitz,${ }^{8}$ B. Serfass, ${ }^{8}$ K. M. Sundqvist, ${ }^{8}$ J-P. F. Thompson, ${ }^{1}$ \\ G. Wang, ${ }^{12,2}$ S. Yellin, ${ }^{7,9}$ J. Yoo, ${ }^{3}$ and B. A. Young ${ }^{5}$
}

(CDMS Collaboration)

\author{
${ }^{1}$ Department of Physics, Brown University, Providence, Rhode Island 02912, USA \\ ${ }^{2}$ Department of Physics, Case Western Reserve University, Cleveland, Ohio 44106, USA \\ ${ }^{3}$ Fermi National Accelerator Laboratory, Batavia, Illinois 60510, USA \\ ${ }^{4}$ Lawrence Berkeley National Laboratory, Berkeley, California 94720, USA \\ ${ }^{5}$ Department of Physics, Santa Clara University, Santa Clara, California 95053, USA \\ ${ }^{6}$ School of Physics \& Astronomy, University of Minnesota, Minneapolis, Minnesota 55455, USA \\ ${ }^{7}$ Department of Physics, Stanford University, Stanford, California 94305, USA \\ ${ }^{8}$ Department of Physics, University of California, Berkeley, California 94720, USA \\ ${ }^{9}$ Department of Physics, University of California, Santa Barbara, California 93106, USA \\ ${ }^{10}$ Department of Physics, University of Colorado at Denver and Health Sciences Center, Denver, Colorado 80217, USA \\ ${ }^{11}$ Department of Physics, University of Florida, Gainesville, Florida 32611, USA \\ ${ }^{12}$ Department of Physics, California Institute of Technology, Pasadena, California 91125, USA
}

(Received 9 September 2005; published 3 January 2006)

\begin{abstract}
We report new results from the Cryogenic Dark Matter Search (CDMS II) at the Soudan Underground Laboratory. Two towers, each consisting of six detectors, were operated for 74.5 live days, giving spectrum-weighted exposures of $34(12) \mathrm{kg} \mathrm{d}$ for the $\mathrm{Ge}(\mathrm{Si})$ targets after cuts, averaged over recoil energies $10-100 \mathrm{keV}$ for a weakly interacting massive particle (WIMP) mass of $60 \mathrm{GeV} / \mathrm{c}^{2}$. A blind analysis was conducted, incorporating improved techniques for rejecting surface events. No WIMP signal exceeding expected backgrounds was observed. When combined with our previous results from Soudan, the $90 \%$ C.L. upper limit on the spin-independent WIMP-nucleon cross section is $1.6 \times 10^{-43} \mathrm{~cm}^{2}$ from $\mathrm{Ge}$ and $3 \times 10^{-42} \mathrm{~cm}^{2}$ from $\mathrm{Si}$, for a WIMP mass of $60 \mathrm{GeV} / c^{2}$. The combined limit from $\mathrm{Ge}(\mathrm{Si})$ is a factor of 2.5 (10) lower than our previous results and constrains predictions of supersymmetric models.
\end{abstract}

DOI: $10.1103 /$ PhysRevLett.96.011302

PACS numbers: 95.35.+d, 14.80.Ly

One-quarter of the energy density of the universe consists of nonbaryonic dark matter [1], which is gravitationally clustered in halos surrounding visible galaxies [2]. The weakly interacting massive particle (WIMP) [3], a dark matter candidate, arises independently from considerations of big bang cosmology and from supersymmetric phenomenology, where the neutralino can be a WIMP [4,5]. A WIMP has a scattering cross section with an atomic nucleus characteristic of the weak interaction and a mass comparable to that of an atomic nucleus.

The Cryogenic Dark Matter Search (CDMS II) experiment $[6,7]$ is designed to detect atomic nuclei in germanium $(\mathrm{Ge})$ and silicon ( $\mathrm{Si}$ ) crystals that have been scattered by incident WIMPs. WIMP events are expected to have recoil energies of a few tens of $\mathrm{keV}$ and rates $\lesssim 1$ event $\mathrm{kg}^{-1} \mathrm{~d}^{-1}$ [5,8]. The CDMS II search is most sensitive to spin-independent (SI) WIMP-nucleon scattering amplitudes [9]. Coherent superposition of SI ampli- tudes gives Ge better sensitivity than $\mathrm{Si}$, except for small WIMP masses, where kinematics increase the Si sensitivity. In this Letter we interpret our data with the SI ansatz. Another paper describes a spin-dependent interpretation of our data [10].

The CDMS II apparatus in the Soudan Underground Laboratory has been described elsewhere [6,7]. At the experiment's core, $Z$ (depth)-sensitive ionization and phonon detectors (ZIPs) measure the ionization and athermal phonon signals caused by recoiling particles in $\mathrm{Ge}$ and $\mathrm{Si}$ crystals $[7,11]$. We report new results from the most recent CDMS data run collected between March 25 and August 8, 2004 , consisting of 74.5 live days. Six Ge (250 g each) and six Si (100 g each) ZIPs were operated in two vertical stacks ("towers") at a temperature of $50 \mathrm{mK}$. This Letter includes the first data from tower 2 , which contains four $\mathrm{Si}$ and two Ge ZIPs. Improvements made since the previous run $[6,7]$ include a reduction of electrical noise and more 
frequent infrared illumination to clear the crystals of spacetrapped charge.

The ZIP detector provides event-by-event discrimination of nuclear recoils from the dominant background of electron recoils. The ratio of ionization energy to phonon energy ("ionization yield") is $\sim 0.3$ for nuclear recoils [12], where yield is $\sim 1$ for electron recoils caused by photons (see Fig. 1). Electron recoils near the detector surface suffer from poor ionization collection and can mimic the ionization yield of nuclear recoils that occur throughout a detector. Each ZIP's phonon sensors are divided into four quadrants. Timing and signal amplitude comparisons among the quadrants provide discrimination against electron recoils, particularly those near the surface. Also, surface electron recoils often deposit energy in adjacent ZIPs within a tower, causing multiple-detector events. Energy from a WIMP recoil would be contained in one detector.

To avoid bias, we performed blind analyses. Events in and near the signal region were removed from WIMPsearch data sets, or "masked" [13]. The cuts that define a signal were determined using calibration data from ${ }^{133} \mathrm{Ba}$ and ${ }^{252} \mathrm{Cf}$ sources and from the nonmasked WIMP-search data. Seven million electron recoils were collected using a ${ }^{133} \mathrm{Ba}$ source of gamma rays, exceeding the comparable WIMP-search data by a factor of 20 . Half of the ${ }^{133} \mathrm{Ba}$ data were used to define analysis cuts and the other half to test

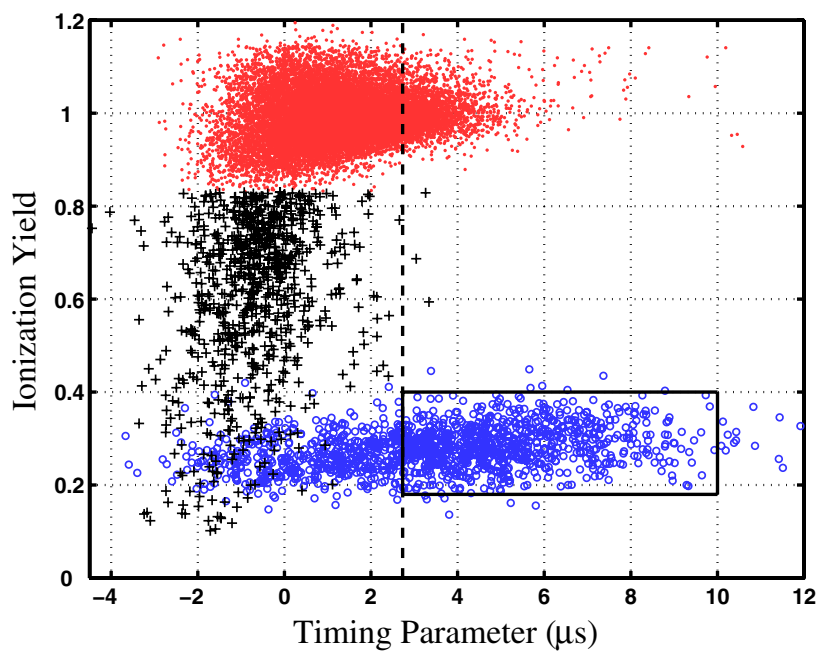

FIG. 1 (color online). Ionization yield versus timing parameter (see text) for calibration data in $\mathrm{T} 2 \mathrm{Z} 3(\mathrm{Ge})$, with recoil energies in the range 10-100 keV. Typical bulk electron recoils from the ${ }^{133} \mathrm{Ba}$ source of gamma rays (dots, light red) have yield near unity. Low-yield ${ }^{133} \mathrm{Ba}$ events (+, black), attributed to surface electron recoils, have small values of timing parameter, allowing discrimination from neutron-induced nuclear recoils from ${ }^{252} \mathrm{Cf}$ $(\mathrm{O}$, medium blue), which show a wide range of timing parameter values. The vertical dashed line shows the minimum timing parameter allowed for WIMP candidates, and the box shows the approximate signal region, which is, in fact, weakly energy dependent. cut definitions and estimate expected backgrounds. The detector response to nuclear recoils was characterized with $10^{4}$ neutrons from a ${ }^{252} \mathrm{Cf}$ source, collected in four separate, several-hour periods.

Data from the ${ }^{133} \mathrm{Ba}$ source were used to monitor detector stability and to characterize detector performance. One Ge detector in tower 2, ZIP 5 [T2Z5(Ge)], had a spatial region of abnormal ionization response that was excluded from analysis. The Si detector T1Z6, known to be contaminated with ${ }^{14} \mathrm{C}$, a beta emitter, was entirely excluded, as were detectors $\mathrm{T} 1 \mathrm{Z} 1(\mathrm{Ge})$ and $\mathrm{T} 2 \mathrm{Z} 1(\mathrm{Si})$ due to poor phonon sensor performance. We therefore report results from $5 \mathrm{Ge}$ and $4 \mathrm{Si} \mathrm{ZIPs}$, chosen before unmasking the WIMP signal region.

Most event selection criteria, based on quantities from the four phonon and two ionization pulses from each detector, are similar to those described in our previous reports $[6,7]$. However, we completed five distinct analyses [14] focused on improving existing methods and developing new techniques to reject surface electron recoils. Events with low ionization yield in the ${ }^{133} \mathrm{Ba}$ calibration data, which are from surface electron recoils, were used to develop rejection criteria. All cuts were frozen prior to unmasking the signal region.

Phonon pulses from surface recoils are more prompt than those from recoils in the detector bulk. Two timing quantities in the quadrant with the largest phonon signal, or "local quadrant," are particularly powerful: the time delay of the phonon signal relative to the fast ionization signal, and the phonon pulse rise time [6,15]. For the first and simplest of the five analysis techniques, the sum of delay and rise time forms a timing parameter, after energy corrections to delay and rise time are applied to achieve an energy-independent distribution. Figure 1 shows the ionization yield versus this timing parameter for a typical $\mathrm{Ge}$ detector. The ${ }^{133} \mathrm{Ba}$ calibration events with yields of 0.1 to 0.8 , identified as surface electron recoils, show a smaller timing parameter than most nuclear recoils induced by the ${ }^{252} \mathrm{Cf}$ source.

For data from the Ge detectors, we require that candidates for WIMP-induced nuclear recoil exceed a minimum value for this timing parameter (see Fig. 2, upper right). Because this "timing parameter" analysis is simple and robust, we agreed before unmasking to report its results. The expected sensitivities computed for the four more advanced analyses described below were all within $\pm 20 \%$ of that computed for this technique.

Two of the four advanced analysis techniques evaluate the goodness of fit, $\chi^{2}$, for surface electron versus bulk nuclear recoil hypotheses. These methods use three variables including their correlations: time delay, rise time, and "partition." The partition parameter, a measure of energy distribution among the phonon quadrants, is defined as the ratio of phonon energy in the local quadrant to that in the diagonal quadrant. The difference in $\chi^{2}$ between surface 

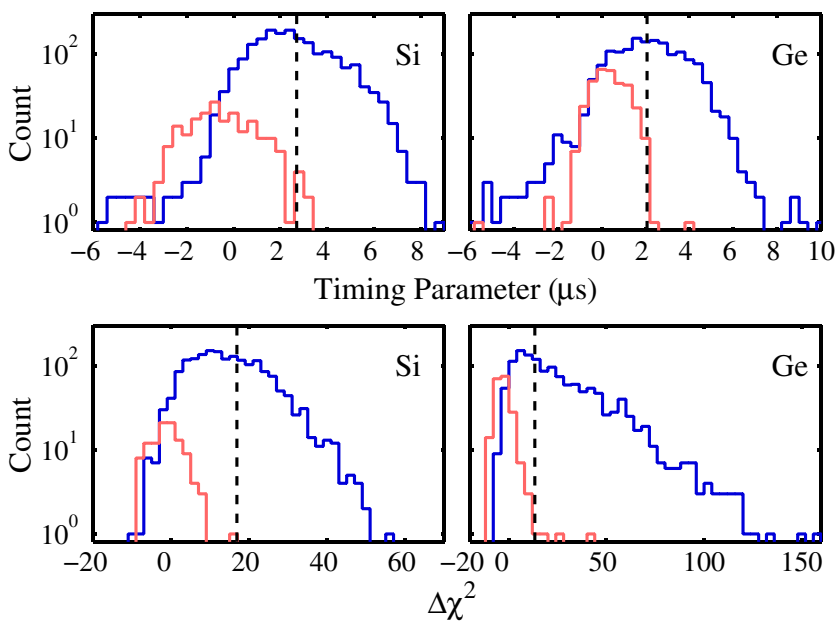

FIG. 2 (color online). Variables used to reject surface electron recoils (7-100 keV), for data from T2Z4 (Si, left) and T1Z2 (Ge, right), for the timing parameter (upper) and $\Delta \chi^{2}$ (lower). Light (red) lines show distributions of low-yield electron recoils from the ${ }^{133} \mathrm{Ba}$ source; dark (blue) lines show distributions of nuclear recoils from the ${ }^{252} \mathrm{Cf}$ source. Dashed lines indicate the minimum values for acceptable WIMP candidates. A cut on the timing parameter is used for the Ge detectors; a cut on $\Delta \chi^{2}$ is used for the Si detectors.

electron and bulk nuclear recoil hypotheses, $\Delta \chi^{2}$, is used as the principal discrimination parameter in these analyses. One method uses energy-dependent variances and the other energy-independent variances.

For the Si detectors, we decided, after unmasking but based only on calibration and multiple-detector events, to use the energy-dependent $\chi^{2}$ technique, since it has the best expected sensitivity to a nuclear recoil signal from low-mass WIMPs of any of our five methods. This analysis technique is 4 times as sensitive as the timing parameter analysis for low-mass WIMPs. Before unmasking, we set the minimum requirement on the $\Delta \chi^{2}$ for surface electron recoil rejection (see Fig. 2, lower left). These criteria are used for our WIMP-search results for Si.

The two remaining analysis techniques confirm the robustness of our $\mathrm{Ge}$ and $\mathrm{Si}$ results. One technique combines delay, rise time, and partition in a neural net analysis, and the other technique exploits additional information from the fitted signal pulses to reconstruct recoil position and type. These two and the $\chi^{2}$ techniques promise further improvements in sensitivity for the larger exposures planned in our future runs, beyond the improvements already demonstrated in Fig. 2.

Upon unmasking the Ge data, one candidate with $10.5 \mathrm{keV}$ recoil energy was found to pass all cuts in the timing parameter analysis. Unmasking the Si data revealed that no events passed all cuts. Figure 3 shows the unmasked data in ionization yield versus recoil energy; the data before and after application of the cut intended to reject surface electron recoils are shown. All analysis techniques showed consistent results.

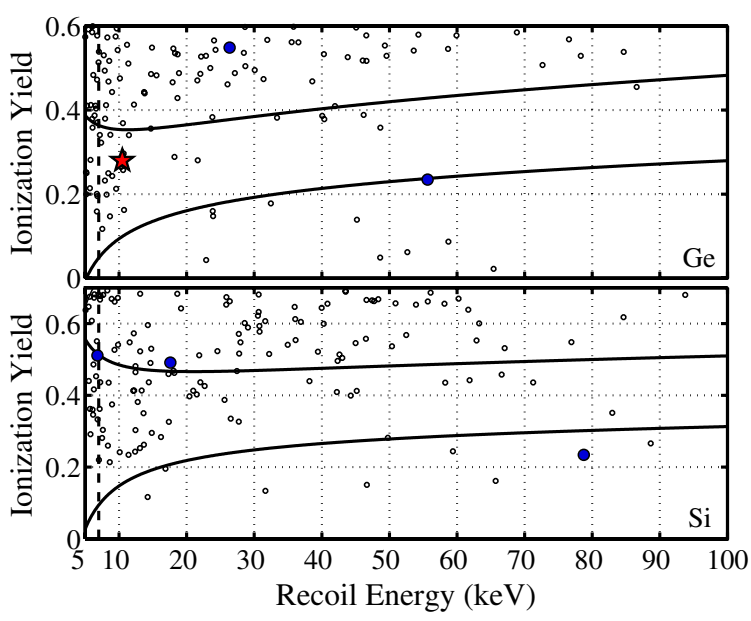

FIG. 3 (color online). Ionization yield versus recoil energy for events in all Ge detectors (upper) and all $\mathrm{Si}$ detectors (lower) passing initial data selection cuts prior to applying the surface electron recoil rejection cut. The signal region consists of recoil energies exceeding $7 \mathrm{keV}$, shown with a vertical dashed line, and yields between the curved lines defined with recoils induced by the ${ }^{252} \mathrm{Cf}$ source while WIMP-search data were still masked. Below $7 \mathrm{keV}$, separation between nuclear and electron recoils becomes poor. Events passing the surface electron recoil cut are a star (red) inside the signal region and dark filled circles (blue) outside. Bulk electron recoils with yield near unity are above the vertical scale limits.

After unmasking the Ge data, we realized that the candidate occurred in a detector during an interval of time when that detector suffered inefficient ionization collection. This defect by itself would prevent us from claiming the event was evidence for a WIMP-induced nuclear recoil. The candidate is also consistent with the rate of expected background. Although we do not claim this event as a nuclear recoil from a WIMP, we do include it in setting limits on the WIMP-nucleon cross section.

The expected surface-event backgrounds are estimated by multiplying two factors. The first factor is the number of events in the signal region as obtained upon unmasking, before surface-event cuts are applied. The second factor is the fraction of surface events expected to pass the surfaceevent cut. This fraction may be estimated from the actual passing fraction of other low-yield events similar to the single-detector event background. An estimate using the passing fraction from ${ }^{133} \mathrm{Ba}$ data indicated a surface-event background of $0.13 \pm 0.05(0.9 \pm 0.4)$ events for $\mathrm{Ge}(\mathrm{Si})$. However, we decided before unmasking to base our background estimate on the passing fraction of WIMP-search multiple-detector events in a wide low-yield region. The number of surface events expected to pass the surfaceevent cuts is $0.4 \pm 0.2$ (stat) \pm 0.2 (syst) between 10 $100 \mathrm{keV}$ in $\mathrm{Ge}$ and $1.2 \pm 0.6$ (stat) \pm 0.2 (syst) between $7-100 \mathrm{keV}$ in Si. Evidence suggests that beta decays of radioactive nuclides distributed across the detectors cause most surface electron recoils in the WIMP-search data. The 


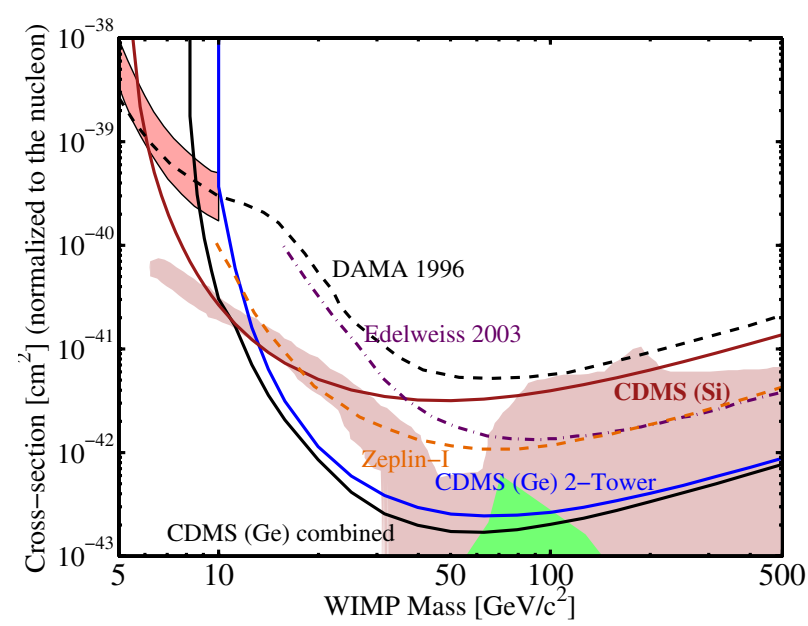

FIG. 4 (color online). WIMP-nucleon cross-section upper limits (90\% C.L.) versus WIMP mass. The upper CDMS Ge curve uses data from only the current run; the lower Ge curve results from data from the current and previous runs [6]. Supersymmetric models allow the largest shaded region [17], and the smaller shaded (green) region [18]. The shaded region in the upper left (see text) is from DAMA [19], and experimental limits are from DAMA [20], EDELWEISS [21], and ZEPLIN [22].

spatial distribution of these contaminants differs from that of surface electron recoils from the ${ }^{133} \mathrm{Ba}$ source, which might explain the difference in estimated background levels. From simulation methods reported in [7], the expected background due to cosmogenic neutrons that escape our muon veto is 0.06 events in Ge and 0.05 events in Si.

Figure 4 shows the upper limits on WIMP-nucleon cross sections calculated from the Ge and $\mathrm{Si}$ analyses reported here using standard assumptions for the galactic halo [8]. For the upper Ge limit, data between $10-100 \mathrm{keV}$ from this run are used. Also shown is the combined limit obtained from this report and our nonblind result from the previous run $[6,7]$. For the combined Ge limit, we have included data in the 7-10 keV interval of recoil energy from the run reported here [16]. The combined result for Ge limits the WIMP-nucleon cross section to $<1.6 \times 10^{-43} \mathrm{~cm}^{2}$ at the $90 \%$ C.L. at a WIMP mass of $60 \mathrm{GeV} / c^{2}$, a factor of 2.5 below our previously published limits. This new Ge limit constrains some minimal supersymmetric (MSSM) parameter space [17] and for the first time excludes some parameter space relevant to constrained models (CMSSM) [18].

The Si limit in Fig. 4 is based on standard halo assumptions using $\mathrm{Si}$ data from $7-100 \mathrm{keV}$ in this run. The $\mathrm{Si}$ result limits the WIMP-nucleon cross section to $<3 \times$ $10^{-42} \mathrm{~cm}^{2}$ at the $90 \%$ C.L. at a WIMP mass of $60 \mathrm{GeV} / c^{2}$. This Si result excludes new parameter space for low-mass WIMPs, including a region compatible with interpretation of the DAMA signal (2-6 and 6-14 keVee bins) as scattering on $\mathrm{Na}[19]$.
This work is supported by the National Science Foundation under Grants No. AST-9978911 and No. PHY-9722414, by the Department of Energy under Contracts No. DE-AC03-76SF00098, No. DE-FG0390ER40569, No. DE-FG03-91ER40618, No. DE-FG0294ER40823, and by Fermilab, operated by the Universities Research Association, Inc., under Contract No. DEAC02-76CH03000 with the Department of Energy. The ZIP detectors were fabricated in the Stanford Nanofabrication Facility operated under NSF.

*Deceased.

[1] D. N. Spergel et al. (WMAP Collaboration), Astrophys. J. Suppl. Ser. 148, 175 (2003); M. Tegmark et al. (SDSS Collaboration), Phys. Rev. D 69, 103501 (2004).

[2] P. Salucci and A. Borriello, Lect. Notes Phys. 616, 66 (2003); T. Broadhurst et al., Astrophys. J. 621, 53 (2005).

[3] G. Steigman and M. S. Turner, Nucl. Phys. B253, 375 (1985).

[4] B. W. Lee and S. Weinberg, Phys. Rev. Lett. 39, 165 (1977); S. Weinberg, Phys. Rev. Lett. 48, 1303 (1982).

[5] G. Jungman, M. Kamionkowski, and K. Griest, Phys. Rep. 267, 195 (1996); G. Bertone, D. Hooper, and J. Silk, Phys. Rep. 405, 279 (2005).

[6] D. S. Akerib et al. (CDMS Collaboration), Phys. Rev. Lett. 93, 211301 (2004).

[7] D. S. Akerib et al. (CDMS Collaboration), Phys. Rev. D 72, 052009 (2005).

[8] J. D. Lewin and P. F. Smith, Astropart. Phys. 6, 87 (1996).

[9] A Majorana neutralino undergoes a scalar interaction of roughly equal strength for neutrons and protons; see K. Griest, Phys. Rev. D 38, 2357 (1988). The SI scattering amplitude is generally sensitive to scalar, vector, and tensor interactions of a spin-1/2 WIMP; see A. Kurylov and M. Kamionkowski, Phys. Rev. D 69, 063503 (2004).

[10] D. S. Akerib et al. (CDMS Collaboration), Phys. Rev. D (to be published).

[11] K. D. Irwin et al., Rev. Sci. Instrum. 66, 5322 (1995); T. Saab et al., AIP Conf. Proc. 605, 497 (2002).

[12] T. Shutt et al., Phys. Rev. Lett. 69, 3425 (1992).

[13] Although software safeguards to enforce the blinding scheme did not work as intended, the blinding policy itself remained in effect, and we exercised care not to obtain any information from inside the WIMP-search signal region. We believe the cuts were developed fully blind.

[14] CDMS Collaboration (to be published); theses and notes at http://cdms.berkeley.edu/Dissertations.

[15] R. M. Clarke et al., Appl. Phys. Lett. 76, 2958 (2000).

[16] Cuts for 7-10 keV interval of recoil energy were frozen before data were unmasked. The decision to report these data was made after the unmasking. Inclusion of this interval increases the expected background by $0.1 \pm$ 0.02 (stat) events and lowers the limit on the WIMPnucleon cross section only for WIMP masses in the $8-11 \mathrm{GeV} / c^{2}$ interval. No candidate events were found in the 7-10 keV interval of recoil energy.

[17] A. Bottino et al., Phys. Rev. D 69, 037302 (2004).

[18] J. Ellis et al., Phys. Rev. D 71, 095007 (2005). 
[19] We show the $90 \%$ allowed region from Fig. 2(a) of P. Gondolo and G. Gelmini, Phys. Rev. D 71, 123520 (2005).

[20] R. Bernabei et al., Phys. Lett. B 389, 757 (1996).
[21] V. Sanglard et al. (EDELWEISS Collaboration), Phys. Rev. D 71, 122002 (2005).

[22] G.J. Alner et al. (UK Dark Matter Collaboration), Astropart. Phys. 23, 444 (2005). 